\title{
Guanidinium Cation
}

National Cancer Institute

\section{Source}

National Cancer Institute. Guanidinium Cation. NCI Thesaurus. Code C1375.

The cationic form and conjug ate acid of the strong ly basic compound guanidine. 